\section{Life and times}

THE recovery of no fewer than eleven kinds of filamentous microfossil from 3,465-million-year-old rocks in Western Australia (J. W. Schopf Science 260 , $640-646 ; 1993)$, shows that life was not only in existence a few hundred million years after the planet formed, but was thriving: rarely does one find single fossils of this antiquity, let alone entire communities. The fossils represent bacteria and presumably photosynthetic (and therefore oxygenproducing) cyanobacteria little different from those a billion years younger. But, as Schopf asks, if such diverse communities were present at this early date, why did their members remain essentially unchanged for so long?

\section{Waste disposal}

THE growing end of a neuron receives the necessary materials by axonal transport from the cell body. The metabolic products of the activity at the growth cone come back down the axon by retrograde transport and home in on lysosomes in the cell body, or in some cases are processed by proteases in the cytosol. P. J. Hollenbeck now illuminates the mechanisms by which the unwanted proteins are despatched from the growth cone (J. Cell Biol. 121, 305-315; 1993). The vehicles that carry them back, he finds, are membrane-encased vacuoles, which take up fluorescent dextran, introduced into the cell, and convey it to the lysosomes in the cell body. If the neuron is allowed to take up similarly labelled materials from the outside world by endocytosis at the growth cone, all the fluorescence again appears in the same kind of vacuole, travelling down the axon. This is a finely tuned mechanism for responding to the imperatives of the law of conservation of mass.

\section{Silt decision}

THE number of games that can be played to illustrate wave-particle duality is proliferating. Besides diffracting light through material slits (Young's slits), one can diffract atoms through gratings composed of immaterial light waves, atoms through slits composed of other atoms and, now, light off a pair of atoms suspended in a vacuum in a magnetic field (U. Eichmann et al. Phys. Rev. Lett. 70, 2359-2362; 1993). The alert might recall that various groups have made unusual, tenuous 'crystals' out of small numbers of ions suspended in a magnetic field; the pair of mercury ions in the new experiment constitutes the simplest such crystal. So the diffraction of laser photons becomes a novel form of Bragg reflection, which effect showed originally that $\mathrm{X}$-rays are

electromagnetic waves and that atoms in solids line up in ordered arrays. Such is progress.

\title{
Planet X: a myth exposed
}

\section{Gerald D. Quinlan}

THERE is no reason for the planetary system to end at Pluto. Astronomers have long debated the possibility of a more-distant tenth planet, usually referred to as Planet $X$. But the scant dynamical evidence requiring the existence of this planet is largely refuted in a new analysis by Myles Standish of the Jet Propulsion Laboratory ${ }^{1}$.

The history of the hypothetical tenth planet begins with the discovery of Neptune $^{2}$. By the late 1830 s the existence of this unseen planet had become undeniable because of discrepancies approaching one arcminute between the observed and computed orbits of Uranus, attributable to gravitational perturbations. The discovery of Neptune in 1846 within one degree of its predicted position was without question the greatest triumph of celestial mechanics at the time. The discovery reduced the discrepancies in Uranus's orbit to the level of arcseconds, comparable with the noise in the observations, but that did not stop some astronomers from predicting a planet beyond Neptune, of a few Earth masses. A long series of searches beginning with Lowell in 1905 culminated with Tombaugh's discovery of Pluto in 1930 .

Pluto appeared tiny right from the start; it is now known to have a mass only 0.2 per cent of that of the Earth, far too small to have caused the irregularities in the orbits of Uranus and Neptune attributed to Planet X. A dedicated group of astronomers have maintained that a tenth planet is required. Some have proposed other reasons for Planet $\mathrm{X}$, blaming it for causing periodic comet showers and mass extinctions on Earth, or for stealing Pluto from Neptune and reversing the motion of Neptune's satellite Triton. Observational surveys by Tombaugh, Kowal and the Infrared Astronomical Satellite (IRAS) would almost certainly have revealed another Pluto or larger planet, but their failure to do so has not stopped many predictions from being made ${ }^{2,3}$, including two more in just the past year ${ }^{4,5}$.

Standish shows in his new work ${ }^{1}$ that the dynamical evidence for Planet $\mathrm{X}$ is readily explained by uncertainties in planetary ephemerides. The construction of an ephemeris involves processing and evaluating the wide variety of data on planetary positions, choosing a dynamical model for the Solar System, and varying the adjustable parameters to give computed orbits that best fit the observations. Planet X's supporters point to three ephemeris difficulties suggesting a tenth planet: the Uranus observations cannot all be made to fit and the residuals show systematic trends; a few known sightings of Neptune before its identity was established are difficult or impossible to fit; and predictions of Neptune's orbit have tended to be inaccurate. Standish examines the first two difficulties; the third is not surprising given Neptune's long orbital period, 165 years (so that not even one orbit has been completed since it was discovered).

The Uranus difficulties result mainly from the use of an incorrect mass for Neptune. The masses of the jovian planets are now known accurately from the Voyager missions, but in the past they were not, and much of the speculation on Planet $\mathrm{X}$ has been based on residuals from the Jet Propulsion Laboratory's ephemeris DE200 and its predecessors, which used a Neptune mass in error by 0.5 per cent. Standish has constructed a new ephemeris with the correct mass and finds that this reduces the root-mean-square Uranus residuals by about 20 per cent and, more importantly, removes the systematic trends. There remains a strong positive bias in the right-ascension residuals from 1895-1905, but Standish has traced the majority of these to a single catalogue of the US Naval Office 9-inch transit telescope (data from two other sources do not show this bias); an observational error such as an incorrect equinox correction is a plausible explanation.

Regarding the prediscovery sightings of Neptune, Lalande's in 1795 is said to differ from ephemeris predictions by seven or more arcseconds, but Standish shows by extrapolating two ephemerides backwards that the predictions are uncertain by at least several arcseconds; combined with the scatter in Lalande's observations this makes the discrepancy rather dubious. The discrepancy for Galileo's observation in 1613 is larger if true, but Galileo's notes are ambiguous, and, moreover, Standish and Nobili have found another Neptune observation by Galileo, this one agreeing with ephemeris predictions (personal communication).

Accurate planetary observations being collected today will in time lead to more stringent tests for a tenth planet. These observations are valuable irrespective of Planet $X$, because planetary ephemerides are needed for other purposes such as spacecraft navigation and pulsar data reduction, and are instrumental in precise tests of theories of gravitation. But for now the search for Planet $\mathrm{X}$ is futile.

The search for smaller objects in the 